of metallic platinum used as a standard. The absorption coefficient is shown as a function of the energy $E$ of the ejected photoelectrons. The three characteristic $L$ absorption edges of platinum are apparent. We will be concerned with the fine structure between the $L_{3}$ and $L_{2}$ edges. In Fig. 2, parts (A), (B), and (C) show data on $K^{3} \cdot \chi(K)$ vs $K$ for the platinum standard and the $10 \%$ and $1 \%$ platinum catalysts, respectively, where $K$ is the photoelectron wave vector, equal to $(0.263 E)^{1 / 2}$, and $\chi(K)$ represents the normalized fluctuations in the absorption coefficient. $^{7-9}$ Parts (a), (b), and (c) of Fig. 2 are Fourier transforms of the EXAFS data over the range $2 \leqslant K$ $\leqslant 20$. Peaks are observed at $R=R_{j}-a$, where $R_{j}$ is the distance from a platinum atom to atoms in the $j$ th coordination shell. The displacement $a$ of the peak position from $R_{j}$ arises from the phase shift. ${ }^{8}$ We will consider the main peak at $R$ near $2.64 \AA$, which represents the first coordination shell $(j=1)$. From data on the platinum standard, $a$ is found to be $0.12 \AA$. Within experimental error $( \pm 0.02 \AA), R_{1}$ for the $10 \%$ platinum catalyst is the same as $R_{1}$ for the platinum standard (2.76 $\AA$ ), while for the $1 \%$ Pt catalyst it appears to be about $0.03 \AA$ shorter. The magnitude of the peak varies approximately as $N_{1} / \sigma_{1}^{n}$, where $N_{1}$ is the number of atoms (coordination number) at distance $R_{1}$ from the reference atom, and $\sigma_{1}$ is the root mean square deviation of the distance about $R_{1}$ due to the vibrations of the atoms. ${ }^{7-9}$ The arrows to the right of the main peak for the platinum standard in part a of Fig. 2 indicate the magnitudes of the corresponding peaks for the two catalysts, as obtained from parts $b$ and $c$ of Fig. 2. The magnitude decreases progressively from the metallic platinum standard to the $10 \%$ and $1 \% \mathrm{Pt}$ catalysts, illustrating the decrease in $N_{1} / \sigma_{1}^{n}$ with increasing dispersion of the platinum. On increasing dispersion from a negligible value $(\sim 0.001)$ for the platinum standard to 0.9 for the small platinum clusters in the $1 \% \mathrm{Pt}$ catalyst, the magnitude decreases by a factor of 3.4.

In low energy electron scattering studies on platinum, Lyon and Somorjai ${ }^{10}$ have found the root mean square displacement of the surface atoms to be clearly higher than that of the atoms in the bulk. Assuming that $\sigma_{1}$ varies similarly, ${ }^{8}$ the value for the $1 \%$ Pt catalyst (which, with a dispersion of 0.9 , consists almost entirely of surface atoms) would be higher than that of the platinum standard. An estimate of $\Delta \sigma_{1}^{2}$ values from our EXAFS data, by a method described previously, ${ }^{8,11}$ supports this assumption. The coordination number $N_{1}$ of the platinum in the $1 \% \mathrm{Pt}$ catalyst is estimated lower than that of the platinum standard by a factor of about 0.6 . Since $N_{1}=12$ ior the platinum standard, we obtain $N_{1}=7$ (with an estimated uncertainty of \pm 2 ) for the $1 \% \mathrm{Pt}$ catalyst.

In summary, the EXAFS data of this investigation illustrate the potential of the method for highly dispersed metal catalysts. The results have a special significance when one considers the technological importance of catalysts of this type.

We thank NSF, ERDA, and the SSRP staff for support for the experiment. Partial support by NSF Grants DMR 74-24261 and CHE 76-11255 for one of us (F.W.L.) is also acknowledged.

${ }^{1}$ J. H. Sinfelt, Prog. Solid State Chem. 10, 55 (1975).

${ }^{2}$ J. H. Sinfelt, Science 195, 641 (1977).

${ }^{3}$ E. B. Prestridge, G. H. Via, and J. H. Sinfelt, J. Catal. 50, 115 (1977).

${ }^{4}$ P. Eisenberger, B. Kincaid, S. Hunter, D. Sayers, E. Stern, and F. W. Lytle, Proc. IV Int. Conf. on Vac. U.V. Radiation Physics, Hamburg, July 22-26, 1974, p. 806.

${ }^{5}$ J. H. Sinfelt, Ann. Rev. Mater. Sci. 2, 641 (1972).

${ }^{6}$ G. R. Wilson and W. K. Hall, J. Catal. 17, $190(1970)$.

${ }^{7}$ F. W. Lytle, D. Sayers, and E. Stern, Phys. Rev. B 11 , 4825 (1975).

${ }^{8}$ E. Stern, D. Sayers, and F. W. Lytle, Phys. Rev. B 11, 4836 (1975).

${ }^{9}$ D. Sayers, E. Stern, and F. W. Lytle, Phys. Rev. Lett. 35, 584 (1975).

${ }^{10}$ H. B. Lyon and G. A. Somorjai, J. Chem. Phys. 44, 3707 (1966).

${ }^{11}$ D. Sayers, E. Stern, and F. W. Lytle, Phys. Rev. Lett. 35, 584 (1975).

\title{
Model networks of end-linked polydimethylsiloxane chains. II. Viscoelastic losses
}

\author{
J. L. Sullivan a) and J. E. Mark ${ }^{\text {b) }}$ \\ Department of Chemistry, University of Michigan, Ann Arbor, Michigan 48109 \\ P. G. Hampton, Jr. and R. E. Cohen
}

Department of Chemical Engineering, Massachusetts Institute of Technology, Cambridge, Massachusetts 02139

(Received 21 June 1977)

Rubberlike networks are usually prepared from polymers of very high molecular weight by the introduction of cross links at randomly located segments along the chains. They may also be prepared, however, by chem- ically end-linking suitably capped chains of much shorter length. ${ }^{1-3}$ These newer end-linking techniques are of practical importance because the polymers employed are of low viscosity and therefore relatively easy to pro- 
TABLE I. Properties of the end-linked PDMS networks.

\begin{tabular}{lclcl}
\hline \hline Sample & $\begin{array}{l}10^{-3} M_{n} \\
\left(\mathrm{~g} \mathrm{~mol}^{-1}\right)\end{array}$ & $M_{w} / M_{n}$ & $10^{5} \nu / 2 V^{\mathrm{a}}$ & $-\log \tan \delta$ \\
\hline$A$ & 18.5 & 1.87 & 2.62 & 1.23 \\
$A-1$ & 45.0 & 1.34 & 1.07 & 0.716 \\
$A-2$ & 32.9 & 1.19 & 1.47 & 0.981 \\
$A-3$ & 25.6 & 1.26 & 1.89 & 1.21 \\
$A-4$ & 9.50 & 1.93 & 5.11 & 1.92 \\
$B-1$ & 4.70 & 1.88 & 10.3 & 2.26 \\
\hline \hline
\end{tabular}

a) Cross-link density, in mols of cross links per $\mathrm{cm}^{3}$ of network at $25^{\circ} \mathrm{C}$.

cess. They are also of considerable interest scientifically since the uniqueness of the cross-linking process permits the preparation of networks of known average chain length and chain length distribution, with very few imperfections such as dangling chain ends, ${ }^{3}$

Such networks of polydimethylsiloxane (PDMS) have been prepared by end-linking hydroxyl-terminated PDMS chains, in the undiluted state and very nearly stoichiometrically, using a tetrafunctional orthosilicate. Some of the equilibrium elastomeric properties of these networks have been reported, ${ }^{3}$ but their dynamic mechanical properties are entirely unexplored. The present study therefore reports viscoelastic losses of end-linked PDMS networks. Comparison of these results with corresponding results ${ }^{4}$ obtained on the randomly crosslinked networks should provide important information in general on the dynamic mechanical properties and structure of PDMS networks.

The end-linked networks studied are described in the first four columns of Table I. Values of the number-average molecular weight $M_{n}$ were those of the uncrosslinked chains and therefore also those of the network chains upon stoichiometric completion of the cross-linking process. ${ }^{3}$ The same applies to the ratio $M_{w} / M_{n}$ of weight-average to number-average molecular weight, which serves as a measure of the breadth of the molecular weight distribution. ${ }^{5}$ The cross-link density at $25^{\circ} \mathrm{C}$ was calculated from $\nu / 2 V=\rho / 2 M_{n}$, where $\nu / 2$ is the number of cross links $s^{5}$ and $\rho=0.9700 \mathrm{~g} \mathrm{~cm}^{-3}$ is the density of the polymer. Dynamic mechanical properties were measured on the unswollen samples exactly as described previously. ${ }^{4}$ In brief, they were determined in shear on a Rheovibron Viscoelastometer using a frequency of 110 $\mathrm{Hz}$, at $175^{\circ} \mathrm{C}$. Values of the storage modulus ${ }^{6}$ were found to be in good agreement with values ${ }^{3}$ previously obtained from essentially equilibrium measurements on the networks in uniaxial extension.

Previously reported viscoelastic losses, identically obtained on PDMS networks randomly cross linked in the undiluted state by means of $\gamma$-radiation, are given by the open circles in Fig. 1. The viscoelastic losses, represented by $\log \tan \delta$ as is customary, are shown as a function of the cross-linked density as obtained, in this case, from equilibrium stress-strain measurements. ${ }^{4,7}$ The line shown was located by least-squares analysis of these results, along with numerous additional results obtained on PDMS networks randomly cross linked in solution。 ${ }^{4}$ Such losses are generally thought to be due to motions of interchain entanglements. Therefore, the fact that the single line shown gave a good fit of all of the viscoelastic losses suggests that cross linking in solution may not significantly decrease the number of interchain entanglements in the resulting network. An alternative explanation, however, would be that the observed losses are due to dangling chain ends, the lengths of which would of course decrease with increase in crosslink density. The present results on the end-linked networks shown this alternative explanation to be untenable. The end-linked networks have very few such chain ends yet their viscoelastic losses, shown by the filled circles in Fig. 1, are well represented by the line characterizing the randomly cross-linked networks. The fact that the agreement seems to hold for all of the samples further suggests that the viscoelastic losses may not be very dependent on chain-length distribution. Also, there does not seem to be any evidence in these results which would support the suggestion that networks prepared by end linking very short chains are essentially free of entanglements.

For all of the PDMS networks studied to date, the viscoelastic losses seem to depend only on the cross-link density and therefore only on the length of the network chains. More definitive conclusions await additional measurements carried out as a function of frequency on these networks and on other PDMS networks of different cross-link functionality.

It should also be mentioned that if it is is assumed that both randomly cross-linked and end-linked PDMS networks should exhibit the same viscoelastic losses, then the present results give strong support for the use of

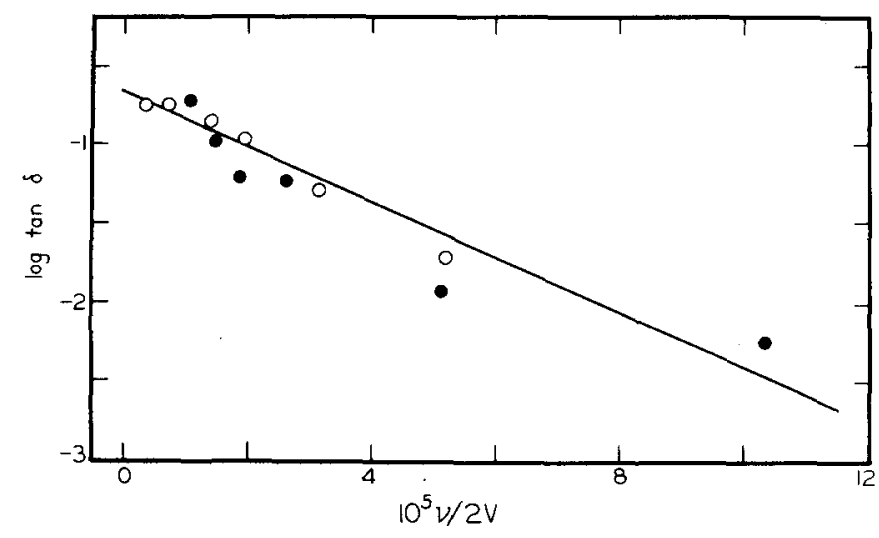

FIG. 1. The logarithm of the viscoelastic loss tangent shown as a function of the cross-link density for tetrafunctionally cross-linked polydimethylsiloxane networks. Measurements were carried out on the networks at $175^{\circ} \mathrm{C}$, in the unswollen state, at a frequency of $110 \mathrm{~Hz}$. The open circles locate results ${ }^{4}$ obtained on networks prepared by randomly crosslinking PDMS chains by means of $\gamma$ radiation. The filled circles locate the present results for networks prepared by chemically end-linking suitably capped PDMS chains. 
$\nu / 2^{8-14}$ rather than $\nu$ for the structure factor in the elastic equation of state derived in the molecular theories of rubberlike elasticity. Superposition of the two sets of results in Fig. 1 requires use of the former factor rather than the latter in calculating the cross-link densities of the randomly cross-linked networks.

It is a pleasure to acknowledge the financial support provided by the Division of Materials Research of the National Science Foundation (Grant DMR 76-20036 to J.E. M.), and by the Donors of the Petroleum Research Fund, administered by the American Chemical Society (Grant 9426-AC-7 to R. E. C.).

a) Present address: Scientific Research Staff, Ford Motor Company, Dearborn, MI 48121.

b) Present address: Department of Chemistry, University of Cincinnati, Cincinnati, OH 45221.
${ }^{1}$ P. Rempp, J. Herz, G. Hild, and C. Picot, Pure Appl. Chem. 43, 77 (1975), and pertinent references cited therein. ${ }^{2}$ M. Morton, L. J. Fetters, J. Inomata, D. C. Rubio, and R. N. Young, Rubber Chem. Technol. 49, 303 (1976).

${ }^{3}$ J. E. Mark and J. L. Sullivan, J. Chem. Phys. 66, 1006 (1977).

${ }^{4}$ R. E. Cohen, S. D. Severson, C. U. Yu, and J. E. Mark, Macromolecules 10, 663 (1977).

${ }^{5}$ P. J. Flory, Principles of Polymer Chemistry (Cornell University, Ithaca, 1953).

${ }^{6} \mathrm{P}$. G. Hampton, Jr., M.S. thesis in Chemical Engineering, Massachusetts Institute of Technology, 1977.

${ }^{7}$ R. M. Johnson and J. E. Mark, Macromolecules 5. 41 (1972).

${ }^{8} \mathrm{H}$. M. James and E, Guth, J. Chem. Phys. 15, 669 (1947).

${ }^{9}$ B. E. Eichinger, Macromolecules 5, $496(1972)$.

${ }^{10} \mathrm{~W}$. W. Graessley, Macromolecules 8, 186, 865 (1975).

${ }^{11}$ K. J. Smith, Jr., and R. J. Gaylord, J. Polym. Sci., Polym. Phys. Ed. 13, 2069 (1975).

${ }^{12}$ R. T. Deam and S. F. Edwards, Philos. Trans. R. Soc. London, Ser. A 280, 317 (1976).

${ }^{13}$ P. J. Flory, Proc. R. Soc. London, Ser。A 351, 351 (1976).

${ }^{14}$ P. J. Flory, J. Chem. Phys. 67, 5720 (1977).

\title{
Pressure-broadened linewidths of $\mathrm{HNO}_{3}^{\text {a) }}$
}

\author{
G. D. T. Tejwani and Edward S. Yeung \\ Ames Laboratory-United States Department of Energy and Department of Chemistry, Iowa State \\ University, Ames, Iowa 50011 \\ (Received 25 October 1977)
}

$\mathrm{HNO}_{3}$ has been detected in the stratosphere through the identification of several of its vibration-rotation bands in the solar spectrum and atmospheric emission spectra. ${ }^{1-9}$ This indicates that $\mathrm{HNO}_{3}$ may play an important role in the photochemistry of the upper atmosphere. ${ }^{10} \mathrm{HNO}_{3}$ is of interest also owing to its significant role in the photochemical reactions of polluted urban atmospheres. ${ }^{11}$ Accurate data on the spectral characteristics of the various molecules found in the atmosphere are necessary for the purpose of detecting and monitoring these minor constituents by infrared techniques. The most useful parameters needed are the vibration-rotation line center frequencies, line strengths, and air-broadened linewidths. The line frequencies are essential for identification of the molecular species while the line strengths and linewidths are necessary in determining the abundance. In this work, we will be mainly concerned with linewidth values.

$\mathrm{HNO}_{3}$ is an asymmetric top molecule with $C_{s}$ symmetry and has very complex vibrational-rotational infrared spectra. There have been no direct measurement of linewidths in any of its bands. We have used the Anderson-Tsao-Curnutte theory of line broadening $^{12,13}$ as expounded by Yamamoto and Aoki ${ }^{14}$ for asymmetric top molecules in our computations for the self-, $\mathrm{N}_{2}-$, and $\mathrm{O}_{2}$-broadened transitions in the $\mathrm{HNO}_{3}$ bands.

$\mathrm{HNO}_{3}$ is a strongly dipolar molecule. ${ }^{15}$ Therefore, just as in the case of formaldehyde, ${ }^{16,17}$ the contribution to the linewidth due to higher order multipole interactions should be completely masked. ${ }^{18}$ We have considered dipole-dipole interaction in $\mathrm{HNO}_{3}$ self -broadening computations. For $\mathrm{N}_{2}$ - and $\mathrm{O}_{2}$-broadened linewidths of $\mathrm{HNO}_{3}$, dipole-quadrupole interaction has been taken into account. The details of computer programs are given in Refs。16 and 19 。

Rotational and centrifugal distortion constants in the ground state of $\mathrm{HNO}_{3}$ have been taken from Kaushik and Venkateswarlu。 ${ }^{20}$ The gound state rotational and centrifugal distortion constants for nitrogen and oxygen were obtained from the pure rotational Raman spectra of these gases. ${ }^{21}$ Kinetic theory collision diameters for $\mathrm{N}_{2}$ and $\mathrm{O}_{2}$ were taken from Svehla. ${ }^{22}$ Kinetic theory collision diameter of $\mathrm{HNO}_{3}$ is also required in the foreign-gas-broadening calculations. It was obtained by using the relations from Ref. 22 and the data on physical properties of nitric acid vapor from Ref. 23. The calculated value for the kinetic theory collision diameter of $\mathrm{HNO}_{3}$ is $3.97 \AA$. The quadrupole moments of $\mathrm{N}_{2}$ and $\mathrm{O}_{2}$ have been taken from the induced birefringence measurements of Buckingham et al. ${ }^{24}$

$\mathrm{HNO}_{3}$ is a planar asymmetric rotor with $c$ axis perpendicular to the plane of the molecule. The permanent dipole moment lies in the plane. Therefore, both $a-$ type and $b$-type selection rules are allowed in seven in- 